\title{
A Systematic Approach to Solving Hatchability and Chick Quality Problems ${ }^{1}$
}

\author{
Gary D. Butcher, DVM, Ph.D. and Amir H. Nilipour, Ph.D. ${ }^{2}$
}

A program involving routine analyses of incubating eggs and hatch residue provides a powerful monitoring and diagnostic tool to breeder, broiler, and hatchery managers. This program would allow managers to stay abreast of many problems with breeder performance, egg management, and broiler grow-out. An organized, scheduled program would verify that the processes of egg production and incubation are being done correctly. Data generated by egg breakout analyses provide information of the flock history, details of breeder and hatchery management, and anticipated early broiler performance. Table 1 provides practical industry standards for the various parameters monitored in an egg breakout program. These standards should be used as a guide for comparison purposes. These parameters were compiled from break-out analyses conducted during a 5-year period from breeder flocks utilized at Group Melo, S.A. in Panama. These data are the average for the 40-week heavy breeder production cycle.
Table 1. Egg break-out data for 40 week production cycle.

\begin{tabular}{|c|c|}
\hline Parameters & $\%$ \\
\hline Hatch & 85 \\
\hline Second quality chicks & 1.0 \\
\hline Dead chicks & 0.25 \\
\hline Pipped eggs & 1.25 \\
\hline Infertiles & 4.50 \\
\hline $1-4$ & 2.50 \\
\hline $5-10$ & 1.25 \\
\hline $11-17$ & 1.25 \\
\hline $18-21$ & 2.50 \\
\hline Contamination & 0.50 \\
\hline Total & 100.00 \\
\hline
\end{tabular}

With optimal breeder farm and hatchery conditions, 85 healthy chicks should be produced from each 100 eggs set. In many companies, hatches of $81-82 \%$ or less are common. In some operations, the number of healthy chicks produced is as low as 70 to 75 chicks per 100 eggs set.

\section{Why Conduct Egg Breakouts?}

Breakout studies are conducted to determine what is "occurring" inside the hatching eggs. The data generated should provide information to direct

1. This document is VM136, one of a series of the Veterinary Medicine-Large Animal Clinical Sciences Department, Florida Cooperative Extension Service, Institute of Food and Agricultural Sciences, University of Florida. Original publication date June 12, 2002. Visit the EDIS Web Site at http://edis.ifas.ufl.edu.

2. Gary D. Butcher, DVM, Ph.D., Diplomate, American College of Poultry Veterinarians, University of Florida College of Veterinary Medicine, Gainesville, FL., Amir H. Nilipour, PhD, Director of Investigation and Quality Assurance, Grupo Melo, S.A., Panama, Republic of Panama 
an investigation as to the likely causes of the problem, so that deficiencies can be corrected. For each parameter in an egg breakout that deviates from the standard in Table 1, an investigation must be conducted to determine the reason. The egg breakout studies must be conducted on a routine, scheduled basis, even when hatch is at an acceptable level. This program reveals if there is a problem at any point in the embryogenesis process, and possibly suggests where corrections can be made proactively. In large modern hatcheries, identifying and correcting even small problems, which result in $0.5 \%$ losses in hatch, can result in considerable savings.

\section{Garbage or Valuable Samples?}

In hatcheries, hatch residues are usually promptly discarded after pulling chicks to permit washing and disinfecting of the hatcher and chick room. That is done to maintain plant sanitation and minimize risk of cross contamination. However, when properly selected, separated, and evaluated, hatch residues provide a valuable and reliable source of information for the breeder farm, hatchery managers, and broiler growers.

\section{Sample Collection Frequency}

Evaluating three trays per breeder farm on a weekly basis is recommended. A representative sample, with each tray separately identified, should include one tray from the top, one from the middle, and one from the bottom of one buggie per farm. The three tray samples must come from the same farm and egg collection day to allow comparisons to be valid.

\section{How to Conduct an Egg Breakout Analysis}

It is a simple procedure that can be learned quickly with training and supervision. It is not necessary to be an embryologist! The requirements include only an interest, organization, clear objectives and appropriate tools.

\section{Plan of Action}

Egg breakout analyses must be conducted in an orderly sequence as follows:
Communication: Maintain channels of communication among breeder, hatchery, and broiler personnel and employ simple language to be understood by all.

Be prepared: The hatchery manager should schedule the farms for the breakout analysis in advance and note the location of the eggs. The data sheets should be available and ready to be filled out with the necessary background information. The minimum data required are age of the flock, breed cross, health status, type of machines used to incubate the eggs, and duration of egg storage.

Vital data: After locating the three preselected trays from a specific breeder flock for breakout analyses, the following information for each tray must be recorded on the data sheets.

- \% hatch for the lot

- \% hatch for the three trays selected

- Total number of eggs per tray selected

- Number of healthy chicks

- Number of dead chicks

- Number of second quality chicks

- Number of exploders

\section{The Egg Breakout Procedure}

From the selected hatch trays, collect the remaining pips, unhatched eggs, and dead chicks. Place the eggs with the large end up in egg flats, and count and record the number of dead chicks. The three flats must be identified as to breeder flock and hatcher tray position. The following steps should be taken to minimize errors and confusion when collecting and analyzing data.

- Count the pips. Remove the shell cap and determine the reason for not hatching. Record observations and comments. To facilitate the process, an assistant may record the findings.

- With a spoon or forceps, gently open remaining eggs at the large end. 
- Remove the eggshell cap and shell membranes with care. The blastoderm or very small embryo may adhere to the inner air cell membrane and be inadvertently discarded. These eggs may be mistakenly classified as infertile.

- After opening and examining the unhatched eggs, tabulate and classify the findings into the following categories: infertiles, embryonic mortalities at 1-7, 8-14 and 15-21 days of incubation and contamination. After classification, discard the eggs. At each stage ensure that all eggs are counted and classified, and the information is recorded on the data form. When conducting the breakout analyses, it is not necessary to remove the egg contents unless there is doubt as to the reason the embryo was unable to hatch. Most commonly, eggs that do not hatch fall into these categories: infertile, deformed, dehydrated, malpositioned, contaminated, oversized embryo, etc.

\section{Determine the Stage of Embryo Development}

This is learned by practice. A common technique is to display a reference poster in the work area which has high quality scale photographs of the embryo and chick at progressing stages of incubation. These posters may be obtained by contacting incubator manufacturing companies. It is also recommended during the learning process to open eggs at various incubation ages to learn how a normal growing embryo develops on a daily basis.

\section{Key distinguishing features:}

- 7-8 days of incubation-- eyes can be distinguished.

-13-14 days-- feathers have started to develop.

- 15 days-- all structures of the embryo have been formed, the embryo is now increasing in size and positioning for hatch.

\section{Fertile or Not Fertile}

In many cases, it is difficult to accurately distinguish among eggs that are fertile, eggs that are infertile or eggs that exhibit positive development when analyzing hatch residue. To increase accuracy of findings, it is recommended to candle the eggs at 9 to 12 days of incubation in the setter, when it is easier to distinguish fertile and infertile. The percent of infertiles and early deads obtained at this stage can be used as a standard at hatch time when the egg breakout is conducted for the same batch.

\section{Establish Standard}

After conducting break-out analyses at an operation and developing a data base, standards for various categories may be generated based on local factors such as breed types, fertile egg source, and feeding and incubation equipment. In hatcheries, three standard tables are commonly developed that take into account the age of the breeders. This variable has a major impact on percent hatch, infertility, and embryonic mortality. The three age periods are usually 25-35, 36-55 and 56-65 weeks. The most dramatic differences in these tables, based on increasing breeder age, would involve percent infertility, second quality chicks and contamination related to egg shell thinning.

\section{Why is the Percent Hatch Declining?}

Hatchability has been declining over the last several years as reported by hatcheries throughout the world. This decline has been related to the recent emphasis on aggressive genetic selection for the characteristic of breast yield over other criteria. However, some deviations in hatch have nothing to do with genetics, but with how we produce, manage and incubate the eggs. Routine egg breakout analyses provide information to enable a manager to determine where problems are occurring and allow timely resolution. Some common causes of hatch failures are described below. A more detailed listing is available in many textbook and hatchery guides.

- High infertility : due to impotent males, unreceptive females, incorrect male to female ratios, mycotoxins in the feed, sexual maturity delays, feed imbalances, high stocking densities, poor ventilation, overweight breeders, lighting problem, etc. 
- Elevated embryo mortality between 1-4 days of incubation: due to egg management, egg room temperature and relative humidity, duration of egg storage, and sperm age and quality.

- Elevated embryo mortality between 5-18 day of incubation: due to machine management, egg turning, too high or low temperature and relative humidity.

- Increased dead in shells: due to poor ventilation in the hatcher, malpositioned embryos, and poor ambient conditions.

The list of causes for hatch failures can be long at the start. However, if a routine egg breakout program is in place, there is no need for panic. The causes of many problems can be promptly identified and reported, and then changes made to resolve the problem. 